\title{
Multi-Level Genetic Algorithm for the Resource- Constrained Re-Entrant Scheduling Problem in the Flow Shop
}

\author{
Danping Lin ${ }^{1}$, C. K. M Lee ${ }^{1}$ and William $\mathrm{Ho}^{2}$ \\ ${ }^{1}$ Division of Systems and Engineering Management, School of Mechanical and \\ Aerospace Engineering, Nanyang Technological University, Singapore \\ ${ }^{2}$ Operations \& Information Management Group, Aston Business School, Aston University, \\ Aston Triangle, Birmingham B4 \&ET, United Kingdom \\ E-mail: lind0020@e.ntu.edu.sg, ckmlee@ntu.edu.sg,w.ho@aston.au.uk
}

Corresponding author: C.K.M Lee

Present address: Division of Systems and Engineering Management, School of Mechanical and Aerospace Engineering, N3.2-02-07, 50 Nanyang Avenue, Singapore 639798

Phone: 65-67906891 fax: 65-67924062

Abstract - Re-Entrant Flowshop Scheduling Problem (RFSP) is regarded as NP-hard problem and attracted the attention of both researchers and industry. Current approach attempts to minimize the makespan of RFSP without considering the resources constraints and interdependency of re-entrant probability. This paper proposed Multilevel Genetic Algorithm (GA) by including the co-related re-entrant possibility and production mode in multi-level chromosome encoding. Repair operator is incorporated in the Multi-level genetic algorithm so as to revise the infeasible solution by resolving the resource conflict. With the objectives of minimizing the makespan, Multi-level Genetic Algorithm (GA) was proposed and ANOVA is used to fine tune the parameter setting of GA. The experiment shows that the proposed approach is more effective to find the near-optimal schedule than the simulated annealing algorithm for both small-size problem and large-size problem.

Keywords-Resource-constrained, re-entrant, genetic algorithm, multi-level encoding 


\section{INTRODUCTION}

In this paper, a multi-level genetic algorithm is proposed to minimize the makespan for the re-entrant flow shop scheduling problem (RFSP) with dual resource constraints. In the re-entrant flow shop, routes of all jobs are identical, while some jobs will visit certain machines more than once at different stages of processing. The number of the reentrant jobs and the re-entrant frequency may increase or decrease based on the manufacturing or repair process of different products. Each workstation consists of several machines and operators, which is regarded as resource constraints.

A typical example of the re-entrant manufacturing system is the semiconductor manufacturing process, especially wafer fabrication line. In the semiconductor line, chemical material is added to the silicon wafers layer by layer where the wafer needs to repeatedly go through the clean rooms, depending on the manufacturing procedure of the wafer. Another example is the maintenances industry where repair parts will iteratively go through typical operations such as inspection, blasting, welding, grinding, fitting, coating, and packing. Moreover, depending on the repairing technology and product specification, the required production capacity and time would be different. Therefore, an extensive study on re-entrant flow shop is essential for balancing the production efficiency and product quality.

According to Tseng and Chen (2009), a mode is the combination of different resources and/or levels of resource requirements with a specified duration. For many scheduling problem, machines and skilled labor requirements vary at different workstations. The selection of the execution mode will directly influence the production duration and product quality. Therefore, it is critical to determine the execution mode for each task. Take the electro-coating as example, sophisticated machines such as high pressure sprayer have high performance but consume more paint and less prone to rework, while skilled worker may use less paint but more time for the same job and has a higher chance of re-work. Hence, different modes of production (i.e. machine or man) will affect rework probability. Therefore, effective scheduling of high cost labor and machine can provide a higher payoff for enterprises.

The re-entrant flow shop scheduling problem considered here is based on a maintenance company specialised in repairing large and complex industrial parts. The repair parts include a wide range of machinery such as buckets, fuel nozzles, shroud, 
and rotors. Approximately ninety-five percent of repair parts of this company need to go through the blasting, heat treating, welding, alloying, grinding, and inspection procedures. If parts fail in the inspection process or new faults are found, the repair parts need to be return to the previous workstations and rework. As long as rework happens, the due dates of the re-entrant jobs are becoming tighter. Moreover, as the repairing execution mode is optional, the corresponding technology and standard will differ. Thus the machine time and required operators will vary. The re-entrant probability and the number of re-entrant jobs will change accordingly. Therefore, what choice of execution mode can affect the overall makespan.

Prior to the problem formulation and proposed method, we will review the related literature in Section 2. Section 3 will formulate the problem and Section 4 elaborates the multi-level encoding genetic algorithm with various operators setting. In Section 5, we describe the experiment and analyze the corresponding results. In section 6 , the main explanation of the experimental results is further discussed and finally section 7 summarizes the paper and draws conclusions based on our experimental results.

\section{LITERATURE REVIEW}

Graves et al. (1983) maybe the first work that systematically mentioned the RFSP term which represents the scheduling problems happened in the shop area with a sequence of operations are processed by machines. Based on the re-entrant characteristics, some jobs would visit certain machines more than once before completion but with the same routing. When each machine processes the set of all jobs in the same order, this problem evolves into the re-entrant permutation flow shop scheduling problem (RPFSP), which is the special case of RFSP.

However this problem was only studied rigorously since 2000 with the demanding manufacturing requirement from the semiconductor industries or integrated circuit fabrication or thin film transistor liquid crystal display process. Exact method, heuristic and meta-heuristic methods all have been proposed for solving the re-entrant flow shop scheduling problem. However, the comprehensive analysis of this problem is not done till the review paper of Lin and Lee (2010) which classifies the re-entrant problems and analyzes the optimization methods of re-entrant manufacturing scheduling. 
Mostly exact methods formulate the re-entrant flow shop scheduling problem as the integer programming problem and simulate the efficiency of the new proposed approaches. However, the computational complexity is high as long run time is used to narrow down the space within the constraints. The referenced work can be found from many studies (Chen and Pan, 2006; Choi and Kim, 2007; Demirkol and Uzsoy, 2000; Haitham and Ruml, 2006; Jiang and Tang, 2008; Kaihara et al., 2010; Nielsen, 2004; Odrey et al., 2001; Pan and Chen, 2003; Park et al., 2000; Scholz-Reiter et al., 2010; Yang et al., 2008).

Heuristic techniques such as dispatching rules and constructive heuristics have also been applied to the re-entrant flow shop scheduling problem. The dispatching rules are applied in the semiconductor industries by testing various experiments for the simulation models (Cigolini et al., 1999; El-Khouly et al., 2009; Perkins and Kumar, 1995). Though these heuristic methods produce good quality solutions, they are not extensively used during the period of 2000-2007 as the running time are often large and increase rapidly with size of the problem.

Meta-heuristic, especially genetic algorithm (GA), evolved to be the new trend for the reentrant flow shop scheduling problem. GA gets the edges over other techniques in the area of large-scale complexity like the very large scale integrated (VLSI) circuit manufacturing or repairing industries which are highly re-entrant, and involves hundreds of machines, restrictions and processing steps. Therefore, (Chen et al., 2008a; Hwang and Sun, 1998; Lee and Lin, 2010; Liu, 2010; Rau and Cho, 2009) used GA to solve the re-entrant problem and mainly focus their objectives on minimizing the makespan. Another meta-heuristic method such as tabu search has been used. In the hybridized tabu search (HTS) model of Chen et al., (2008b), the proposed HTS is compared with pure tabu search and NEH (Nawaz et al., 1983) to find out the optimal makespan for various problem sizes. While in the interactive models of Alfieri (2009), a neighborhood search is started with non-dominated and dominated initial solutions, then weighted function is used to evaluate the best solution that becomes the seed for the successive tabu search iteration. The results are compared with composite priority rule in term of maximum tardiness and total delay which is weighted by the customer priority.

Among all these papers, it is obvious that exact methods and meta-heuristics are the main used tools because efficient and economic computational method is required for 
solving this complex scheduling problem. Moreover, most of them adopted minimizing the makespan as their aims. However, most of the works assumed unlimited buffer or no resource constraint in the processing procedure, which do not represent the real case in the world. Or some papers relaxed these constraints and decomposed the problem into sub-problems. Only the work of Scholz-Eriter et al. (2010) that fully takes the resource constraints into account. In their work, machines and labor resources are taken into consideration, priority rules and rule combinations are simulated both for small problem instances and long term system behavior with a time span of ten years. The results revealed that the performance of priority rules depends on the utilization level of the system, thus a proper control system is crucial to select and combine the priority rules.

There are many papers about the flow shop with constrained resource. Generally these works can be classified into two types based on the objectives. The first type related to allocating and scheduling the limited resource to the flow shop. Daniels et al. (2004) discussed the feasibility and benefits of dynamic assignment of partially flexible labor resources, linked skill matrices with self-buffering production line and eventually proved the benefit of complete flexibility can be achieved by a reasonable small amount of carefully selected partial flexibility. Similarly, in the work of Neubert and Savino (2009), a mathematical model that based on constraint optimization principles is proposed to solve the operator scheduling problem. In their paper, the entity of the scheduling optimization is the human resource too.

The second type of flow shop with limited resource concerns the jobs scheduling problem, while the resource constraints are one of their considerations. The example can be found from Janiak (1988), whose main objective is to minimize the job overall completion time. In his model, the duration of each operation on certain machines is a liner function of the allocated part of a constrained resource. The proposed algorithm is based on disjunctive graph theory and brand \& bound algorithm. However, resource allocation is regarded as one of the concern for scheduling. The similar work has been done by Cheng and Janiak (2000) whose algorithm is based on a branch \& bound algorithm and three approximate algorithms. The experimental results revealed that computation time is dependent on the starting solution and the value of the lower bound. Other related work can be found from Leu and Hwang (2002) who present a GA-based search for a precise flow shop production in consideration of resource constraints and different due days of multiple production package. 
Although there are a lot of research has been done on re-entrant flow shop scheduling problem, according to authors' knowledge, no scholars study the problem that the reentrant probability is connected with the execution mode. In this paper, Multi-level GA is proposed to optimize the resource utilization and minimize the makespan by considering the re-entrant probability of the execution mode.

\section{PROBLEM FORMULATION}

The problem of a re-entrant flow shop scheduling problem can be basically described as $m$ machines in series and $n$ jobs to be processed in a line. Job i has $\mathrm{N}_{\mathrm{i}}$ operations and the processing time of its $\mathrm{j}^{\text {th }}$ operation $\mathrm{P}_{\mathrm{ij}}$ is deterministic and prescribed in advance. For jobí, its $\mathrm{P}_{\mathrm{ij}+1}$ must be initiated right after the completion of $\mathrm{P}_{\mathrm{ij}}$. As part of the flow shop scheduling problem, RFSP follows the same assumptions about the job sequence, machine rules and operating policies (Gupta and Stafford, 2006). To make problem simple, we assume that all re-entrant jobs will go back to the first workstation and virtually go through all the workstation again. But only those workstations in which the reentrant jobs get rework have the processing time and related resource consumption.

Notation

$\mathrm{N}$ : the set of all jobs

$\mathrm{n}$ : the number of jobs

$\mathrm{i}, \mathrm{j}$ : job index

r: re-entrant job index

m: machine index

$\mathrm{M}_{\mathrm{i}}$ : the set of machines to process job $\mathrm{i}$

$\mathrm{E}_{\mathrm{m}}$ : the set of jobs that might be processed on machine $m$

$\mathrm{B}$ : the set of pairs of jobs between which there is precedence relationship, when job i must precedence job j

$\mathrm{p}_{\mathrm{im}}$ : processing time of job $\mathrm{i}$ in machine $m$ 
$\mathrm{t}_{\mathrm{i}}$ : the stating time of jobi

$\mathrm{C}_{\max }$ : the completion time of the last job.

Decision variables:

$\mathrm{x}_{\mathrm{im}}=1$, if operation of job $\mathrm{i}$ is assigned to machinem; 0 , otherwise;

$y_{\mathrm{ijm}}=1$, if operation of job $\mathrm{i}$ and $\mathrm{j}$ are assigned to the same machinem; 0 , otherwise;

$\mathrm{z}_{\mathrm{ijm}}=1$, if operation of job 1 immediately precedes on machinem;

$\mathrm{r}_{\mathrm{imk}}=1$, if operation of job $\mathrm{i}$ is conducted on machine $\mathrm{m}$ with $\mathrm{k}$ resource consumption;

The objective of minimizing the makespan is expressed as

Minimize $\sum_{i_{2}, m=1}^{n+r} p_{i m} x_{i m}$

S. T. $\sum_{m \in M_{i}} x_{i m}=1, \forall i \in N$

$\mathrm{t}_{\mathrm{i}} \geq 0, \forall \mathrm{i} \in \mathrm{N}$

$\mathrm{t}_{\mathrm{i}}+\mathrm{p}_{\mathrm{i}} \leq \mathrm{t}_{\mathrm{i}+1_{\mathrm{a}}} \forall \mathrm{i} \in \mathrm{N}$

$y_{i j m}=y_{j i m}, \forall i, j \in E_{m}, \forall m \in M_{i}$

$y_{i j m} \leq 0.5\left(x_{i m}+x_{j m}\right) \leq y_{i j m}+0.5, \quad \forall i, j \in E_{m}, \forall m \in M_{i}$

$\sum_{\mathrm{j} \in \mathrm{E}_{\mathrm{m}}} \mathrm{Z}_{\mathrm{ijm}} \leq 1, \forall \mathrm{i}, \mathrm{j} \in \mathrm{E}_{\mathrm{m}}, \forall \mathrm{m} \in \mathrm{M}_{\mathrm{i}}$

$\sum_{\mathrm{j} \in \mathrm{E}_{\mathrm{m}}} \mathrm{Z}_{\mathrm{jim}} \leq 1, \forall \mathrm{i}, \mathrm{j} \in \mathrm{E}_{\mathrm{m}}, \forall \mathrm{m} \in \mathrm{M}_{\mathrm{i}}$

$z_{\mathrm{ijm}}+\mathrm{z}_{\mathrm{jim}} \leq 1, \forall \mathrm{i}, \mathrm{j} \in \mathrm{E}_{\mathrm{m}}, \forall \mathrm{m} \in \mathrm{M}_{\mathrm{i}}$

$\mathrm{t}_{\mathrm{i}} \leq \mathrm{t}_{\mathrm{j}}, \forall \mathrm{i}, \mathrm{j} \in \mathrm{B}$

$\sum_{m \in M_{i}} \sum_{i \in N} r_{i m k} x_{i m} \leq R_{k}, \forall i \in N_{y}, \forall m \in M_{i}$ 
$\mathrm{x}_{\mathrm{im}}, \mathrm{y}_{\mathrm{ijm}}, \mathrm{z}_{\mathrm{ijm}}, \mathrm{r}_{\mathrm{imk}}=0$ or $1, \forall \mathrm{i}, \mathrm{j} \in \mathrm{N}, \forall \mathrm{m} \in \mathrm{M}_{\mathrm{i}}$

Eq. (1) minimizes the total makespan of all jobs as the objective. Constraints (2) ensure that each operation must be processed by exactly one machine. Constraints (3) ensure that each operation begins after time zero. Constraints (4) ensure that the order of operation of each job is respected. Constraints (5) and (6) ensure that $y_{i \mathrm{im}}=y_{\mathrm{jim}}=1$ when $x_{i \mathrm{ijm}}=x_{\mathrm{jim}}=1$. Constraints (7) and (8) ensure that each operation has at most one predecessor and successor on machine $m$. Constraints (9) ensure that $\mathrm{z}_{\mathrm{ijm}}$ and $\mathrm{z}_{\mathrm{jim}}$ cannot simultaneously equal to 1 . Constraints (10) determine the set of pairs of jobs between which there is precedence relationship. Constraints (11) promise the consumed resource is within the limitation. Constraints (12) are binary constraints.

\section{MULTI-LEVEL ENCODING GENETIC ALGORITHM}

Flow shop scheduling problem is known as NP-hard. When re-entrant character is added, the complexity increases. Moreover, the problem discussed here has specific feature of interdependency between the execution mode selection and the re-entrant probability. It is inappropriate to isolate these two factors or decompose the problem and optimize the makespan individually. Therefore, the solution should consider the following factors. Firstly, they should be able to balance the re-entrant probability and total flow time at the same time; secondly, they are prone to be stochastic which can bring different combination on the execution mode selection and re-entrant probability; thirdly, they are computational effective.

Based on these considerations of the required techniques, genetic algorithm is the preferred option. First of all, genetic algorithm is probabilistic, not deterministic. It works on a population of possible solutions, thus the searching direction is random and has higher possibility to reach the optimal solution. Secondly, the problem presentation of GA is translated via encoding, the job index can be directly encoded as the chromosome and the sequence of the index is definitely the permutation of the scheduling. Thirdly, the efficiency of the GA has been proved by many other applications, its ability of synchronous exploration of multiple objectives is the most outstanding feature. Therefore, GA is the preferred optimization approach for RFSP. 
In the proposed Multi-level GA, multi-level chromosome representation is used to encode the job scheduling, re-entrant probability and execution mode accordingly. Repair operator is adopted to revise those infeasible solutions as well. The details will be presented in the following subsections.

\subsection{Sketch of Multi-level GA for RFSP with Dual Resource Constraints}

(insert figure 1 here)

Fig. 1. Framework of multi-level GA for resource constrained scheduling.

Fig. 1 gives an overview of the proposed framework. The input to the GA includes the data collection like the resource, technical data, the order arrival date and job due date. The dual resource constraints are the machine and labor, and they are measured in term of hours that a specific resource is required. As machine has its capacity and human needs to on shift, the available machine time and man hour are specified at a given period. The user control parameters are also input to this system. The user control parameters include the machine warm-up time, set up time, experiment time duration, etc. In this structure, the iterative loop of GA is also illustrated in the upper part of Fig. 1.

(insert figure 2 here)

Fig. 2. Pseudocode for the GA.

Fig.2 describes the flow of the GA in term of pseudocode. It is noted that repairing operator is used to modify the infeasible solutions before they are included in the next generation. The repairing operator will check the logic of the re-entrant jobs, and examine whether re-entrant jobs are arranged before the original work or not. If the genome of the re-entrant job is found to be allocated ahead of the original job, the reentrant job will swap the position with the original job.

\subsection{Genetic Algorithm}

\subsubsection{Multi-Level Chromosome Encoding}


A suitable chromosome encoding method must be chosen to ensure the problem's information is well presented and appropriate genetic operators are applied. The application of GA to real-world problems usually follows two main approaches, namely, the use of direct and indirect chromosome representations. According to Topcuoglu et al. (2007): for the direct representation, a chromosome represents a single solution. In the indirect version, one or more decoding algorithms are proposed in order to build the solution by considering the chromosome representation. Even though in the proposed method, multi-level chromosomes contain different information and have different data types, one group of multi-level chromosomes represents one solution. Therefore direct chromosome representation is chosen and each population member is comprised of three levels of strings: the first level is the sorted permutation of jobs, and they are shown in unique index, which is the direct representation of the schedule information; the second level is the related re-entrant possibility of each job per different execution mode, and the re-entrant possibility is represented as binary number; and the third level is the corresponding resource consumption of different execution mode that uses real number. The GA operators are applied independently to the levels of strings. The following sections will detail the implementation.

(insert figure 3 here)

Fig. 3. One-level encoding vs. Multi-level encoding.

Fig. 3 illustrates the example of the multi-level encoding used. There are two types of execution mode, and marked as mode 0 and mode 1 . Different modes consume different amount of machine unit time and labor unit time. Take coating process as example, high-tech machine can spray faster and evenly with more paint while manual work consumes less paint but more time with higher chance of rework. But the welding process is the opposite case. Manual work promises better welding within the tolerance of quality standard, while automatic welding costs more filler material and have higher chance of rework. Therefore, it is assumed that as long as the re-entrant probability exceeds 50 percents, the job is recognized as re-entrant job and will enter the production line again.

Assumed there are 6 jobs need to be scheduled. The first level is six unique indexes for jobs, and the binary numbers in the third level are the selection of the execution mode for each job. Second level contains six percentage numbers which individually represent 
the re-entrant possibilities of each job under the execution modes that shown in the $3^{\text {rd }}$ level. Different execution modes will consume different machine and labor resources. During the encoding, it will randomly choose either mode 0 or mode 1 as its execution mode, thus its re-entrant possibility and corresponding resource consumption will be tracked. Take the Parent 1's execution modes in Fig. 3 as example, it is obvious that the job 1, 3, 5 have the rework probability more than 50 percents, which means all these jobs need to be reworked. The re-entrant jobs will be given other indexes to differentiate with the original job indexes. In this example, the job index with asteroid means the reentrant job. No matter jobs are original jobs or those re-entrant jobs, they should within the overall resource constraints. Therefore, there are nine jobs for Parent 1 to schedule with the aim of minimizing the total makespan.

It is possible to encode the chromosome in one level. However, when the re-entrant number is not fixed such that the length of the chromosome is not fixed, the crossover operator is hard to implement. Because the cutting point may happens to be placed between the job index and the re-entrant possibility. After crossover, the abnormal offspring may be generated. In the one-level encoding way, Fig. 2 shows that after crossover is implemented, the offspring 1 would have 6 jobs with 7 re-entrant possibilities that are against the operational practice. Therefore, multi-level encoding which stores the information independently is more appropriate to present the problem and is easier to carry out the crossover/mutation. The detail will be further illustrated in the Section 4.2.2.

\subsubsection{Crossover and Mutation Operators}

As the multi-level chromosome is encoded with different data type for different levels, the crossover operators are conducted independently for different levels. Take the mentioned six jobs case as example, as shown in Fig. 4, two-point crossover is executed for execution mode in the $3^{\text {rd }}$ level, while the $1^{\text {st }}$ and $2^{\text {nd }}$ level are kept constant. Then the re-entrant possibilities in the $2^{\text {nd }}$ level are moved concomitantly and the values are unchanged. According to the renewed possibilities, the re-entrant jobs are updated and two offspring of the job sequence are generated. After that, two new job sequences will be extracted and implemented crossover again. During the crossover procedure, the job index, reentrant probability, executive mode will be changed correspondingly.

(insert figure 4 here) 
Fig. 4. Crossover procedure.

Mutation procedure is similar to the iterative crossover which starts from the $3^{\text {rd }}$ level and mutation only needs one parent. For the mutation, as the job schedule is limited by the resource constraint, the mutating job position should within some particular range to satisfy the resource constraint. In Fig. 2, take parent 1 as example, the previous feasible execution mode is $\left\{\begin{array}{llllll}0 & 1 & 1 & 0 & 0 & 0\end{array}\right\}$, the mutation operator would randomly change the execution mode of one position. At the same time, it will check whether the new chromosome is still within the resource constraint. If the new chromosome is within the resource constraints, keep the chromosome; otherwise, mutate the genes of chromosome. In this scenario two feasible sequences of execution mode can get: one sequence is $\left\{\begin{array}{llllll}1 & 1 & 1 & 0 & 0 & 0\end{array}\right.$ \} with 3 jobs rework too (job index 1, 3 and 5 need to rework), and another sequence is $\left\{\begin{array}{lllllll}0 & 1 & 1 & 1 & 0 & 0\end{array}\right.$ \} with only two jobs (index 3 and 5) rework. Later the mutation for the job index level is implemented similarly, and the duplicated numbers during the process will be repaired.

\subsubsection{Repairing Operator}

A feasible solution for the resource-constrained re-entrant flow shop scheduling problem must not violate resource constraints and the logic of the reentrance process. During the crossover and mutation procedures, re-entrant jobs assigned in the chromosome during the propagation may be positioned before the original jobs. In this case, repairing operator will check the logic of schedule and swap the positions of re-entrant jobs with the original jobs. Besides, repairing operator will also check the resource constraints.

\subsubsection{Fitness Evaluation}

The objective of the proposed GA is to find the solutions to the re-entrant flow shop scheduling problem that minimize the makespan as well as satisfy the resource constraints. So the fitness function is set the same as the objective function.

\subsubsection{Population Size and Stopping Criteria}

Foregone experiments showed that constant population size and constant number of generations can generate good average results even though the problem size increases. The number of re-entrant jobs is increased correspondingly, which greatly amplifies the problem complexity. Thus, we set the stopping criteria as a maximum of 100 generations 
while the population of each generation (190) is determined by statistical experiments which will be described in the next section. These parameters combination will lead to the best results and the highest degree of robustness.

\section{EXPERIMENTAL RESULTS}

The proposed genetic algorithm method has been implemented in the MATLAB 7.0 on a Pentium 4 3.17GHz PC with 3.25G MB memory. To perform a qualitative assessment of our method, the experiments are tested and compared with the homogeneous algorithm of simulated annealing (SA) algorithm. We used the following procedure to compare the quality of solutions found by our GA. In the first place, the comparison is carried out without GA parameter tuning. After the design of experiment to find out the most influenced factor of the GA parameters, new comparison will be done for larger problem size. The reasons for choosing SA for comparison are: firstly, similar as GA, SA is a probabilistic meta-heuristics, so it owns the same advantages of meta-heuristics. Secondly, SA is more suitable to be used for the discrete search space while GA does not have such preference. When the character of the searching space is unknown like the RFSP here, SA can be a good reference. Therefore, in the experiments, SA is run in the same computer configuration and uses the same objective function. The parameters of SA have been tuned in advance and shown below.

The tested data and the standard instances come from the problem library PSPLIP developed by Kolisch and Sprecher (Kolisch and Sprecher, 1997). The processing time is exponential distributed with the mean of 15 and the re-entrant probability is uniformly distributed. The comparison will include varying problem sizes and solution complexities to study the effectiveness of the proposed method. In the experiments without parameter tuning, the size of the GA population was set as 200 , the crossover probability was 0.8 , and the mutation probability is set as 0.4. Each of the GA runs was stopped after 100 generations. Table 1 summarizes the GA and SA parameters used in the experiments, GA's parameters have not been tuned while SA's parameters are tuned in advance. Since both GA and SA are stochastic algorithms, ten independent runs with different random initial population were performed for different problem sizes. The best solution by each algorithm was recorded and better solution was marked in bold.

Table 1 Parameter setting for GA and SA 
(insert table 1 here)

\subsection{Comparing Genetic Algorithm with Simulated Annealing}

In these tests, we give the mean, standard deviation and best performance referring different problem size respectively. Table 2 shows the solutions found by GA and SA in these ten runs with re-entrant character and resource constraints. Ideally, the GA-based method should converge to the optimal or near-optimal solution in each run. Computing the average of the best solutions found over the ten independent runs provides an indication of the robustness of the search strategy. In ten out of the twelve tests, GA works better than SA. For the two out of twelve tests showing GA is inferior to SA, the differences of the fitness between them are less than $0.3 \%$. This illustrates that the proposed GA approach is able to consistently converge to high-quality solutions in every GA run for different problem sizes.

Table 2 Average performance of GA and SA without GA parameter tuning (insert table 2 here)

\subsection{Identifying Efficient Genetic Algorithm Parameters}

Design of experiments (DOE) was performed to determine the favorable parameters of the GA. In general, if computation time is unlimited, the best results were obtained with large populations. Furthermore, the quality of the population is based on the proper GA parameters setting. Therefore, the combination of GA parameters determined the quality of the solution. In practical cases when the computational time is limited, the selection of appropriate GA parameters and the combination of the parameters are crucial to the search result. The tested parameters are shown in Table 3.

Table 3 Tested parameters for GA

(insert table 3 here)

We found that there are statistically significant differences only for the population size, crossover rate and mutation rate. To further explore the importance of these three factors, full factorial design was replicated 10 times in Table 4. The probabilities of the three factors are all considered at two levels. In Table 5, the F-test results for each problem size are presently respectively. It is obvious that at $99 \%$ confidence interval, 
only the factor A (population size) is considered by the analysis of variance (ANOVA) to have significant influence on the fitness value (makespan).

Table 4 Experimental factors

Table 5 ANOVA results

(insert table 4 and 5 here)

\subsection{Scheduling a Larger Problem Size}

Based on the parameters from the ANOVA test, we applied them into the large problem size tests again, in which the job sizes range from 50 jobs to 200 jobs, with uniformly distributed re-entrant probability. New experiments are tested with four scenarios. As we have two execution modes and two limited resources, four scenarios are setting as:

Scenario 1, resource 1 is kept constant and 10 percents increment for resource 2;

Scenario 2, resource 2 is kept constant and 10 percents increment for resource 1;

Scenario 3, resource 1 is kept constant and 10 percents decrease for resource 2;

Scenario 4, resource 2 is kept constant and 10 percents decrease for resource 1.

Table 6 tabulates the results from the experiments by comparing the makespan of the reentrant lines found by GA with SA. The bold letter indicates the better result and the objective is to minimizing the makespan. From Table 6, around seventy-five percents of all the cases show the proposed multi-level GA finds better solutions than SA. The improvement is about 1 percentage.

Table 6 Comparisons of our GA with SA for larger problem sizes

(insert table 6 here)

\section{DISCUSSION}

The search space of the re-entrant flow shop scheduling problem is large even for reasonably problem size. When the number of the re-entrant job is dynamic, the searching difficulty increases. If the number of re-entrant jobs and re-entrant frequency 
are influenced by the execution mode, and the execution mode is constrained by the resource, the complexity of the problem magnifies. Therefore the scheduling of such problem is complicated in the large job size circumstance.

Exact methods can sometimes solve small instances of a complex problem effectively, but it is unable to solve problems of modest or large size within a reasonable period of time. As mentioned before, re-entrant scheduling problem is a complex problem, the computing time to find the optimal solution would increase with an exponential function of the problem size. Therefore, meta-heuristic methods, which can find a solution that is "good enough" in a computing time that is "small enough", can find a nearly optimal solution with a reasonable computational time compared with exact methods. Generally, meta-heuristics are more flexible in two aspects: firstly, they can be adapted to fit the needs of most real-life optimization problems in terms of solution quality and allowed computing time. Secondly, they do not put any demands on the formulation of the optimization problem. Techniques that use global approach, such as genetic algorithm and simulated annealing algorithm, tend to more suitable for re-entrant scheduling problem. The dependence of the resource constraint and the re-entrant possibility make it necessary to do the execution mode selection and scheduling simultaneously. Therefore, the potential techniques should be able to fulfill this requirement. On the other way, the computational time of exact method is exponential increase as the size of the problem increases, so the exact methods with large runtime are not suitable for practical concern.

GA and SA belong to the meta-heuristic methods which are capable to do the global search and implement stochastic optimization provided that the goal is acceptable in a tolerable amount of time. There are still some differences between them. Simulated annealing algorithm works on a single solution and traverses the search space by applying local transformations to the solution representation. The effectiveness and efficiency of the search mainly depend on whether it can escape the local minima states without losing much computational energy (which is roughly exponential on the energy difference among the surrounding states). Thus the results are not so promising.

On the contrary, GA's global search capability lies on the chromosome representation and search operators. In our proposed GA approach, we use multi-level encoding as the problem-specific representation where each level indicates one subtask of the whole 
work. For the job sequence, we use the permutation representation, where the operations in the chromosomes are topologically sorted. This property is preserved during each crossover or mutation operations by repairing procedure. This repairing procedure, on one hand makes sure the practical concern is taken care. On the other hand it alleviates the computational time spent for the phenotype encoding. Then the most influenced factor of the search operators is found using design of experiment procedure. In addition, ANOVA tests are carried out to obtain the best combination of the parameter setting for following larger problem size experiments. The results confirmed that this kind of encoding method can lead to good convergence with enough diversity of population. Generally, the performance of GA is better and more steady-going.

\section{CONCLUSION}

Efficient design of the re-entrant flow shop scheduling is critical for those semi-conductor industries or repairing companies when facing the resource constraints. Several approaches have been proposed for this problem, most of which ignore the dependency between the re-entrant possibility and execution mode selection by considering the resources constraints. In this paper, we have presented a multi-level GA-based approach for balancing the rework percentage and causing higher resource consumption. In this approach, the multi-level encoding keeps the intact information. On the other hand, fastening the convergence of solution is crucial because the execution mode selection and job scheduling are integrated and optimized together. Experimental results on small problem instances have demonstrated the good performance and consistency of the proposed approach when compared with simulated annealing algorithm. Furthermore a series of experiments have been carried out to determine the most influenced factor for the GA and the best combination of parameters have been examined. Based on the fine-tuned parameters, large problem size testes are executed and GA shows superiority over SA. The contribution of this paper is the presentation of a robust multi-level encoding genetic algorithm for resource-constrained re-entrant scheduling problem in the flow shop. The approach is customizable and flexible so as to provide significant potential for extension to other constrained optimization problems.

\section{REFERENCES}

Alfieri, A., 2009. Workload simulation and optimisation in multi-criteria hybrid flowshop scheduling: a case study. Int. J. Prod. Res. 47, 5129-5145. 
Chen, J.-S., Pan, J.C.-H., 2006. Integer programming models for the re-entrant shop scheduling problems. Eng. Optim. 38, 577-592.

Chen, J.-S., Pan, J.C.-H., Wu, C.-K., 2008a. A hybrid genetic algorithm for the re-entrant flow-shop scheduling problem. Expert Syst.with Appl. 34, 570-577.

Chen, J.-S., Pan, J.C.-H., Wu, C.-K., 2008b. Hybrid tabu search for re-entrant permutation flow-shop scheduling problem. Expert Syst.with Appl. 34, 1924-1930.

Cheng, T.C.E., Janiak, A., 2000. A permutation flow-shop scheduling problem with convex models of operation processing times. Ann. Oper. Res. 96, 39-60.

Choi, S.W., Kim, Y.D., 2007. Minimizing makespan on a two-machine re-entrant flowshop. J. Oper. Res. Soc. 58, 572-581.

Cigolini, R., Comi, A., Micheletti, A., Mp, A., 1999. Implementing new dispatching rules at SGS-Thomson Microelectronics. Prod. Plan. and Control 10, 97-106.

Daniels, R.L., Mazzola, J.B., Shi, D., 2004. Flow shop scheduling with partial resource flexibility. Manag. Sci. 50, 658-669.

Demirkol, E., Uzsoy, R., 2000. Decomposition methods for re-entrant flow shops with sequence-dependent setup times. J. Sched. 3, 155-177.

El-Khouly, I.A., El-Kilany, K.S., El-Sayed, A.E., 2009. Modelling and simulation of reentrant flow shop scheduling: An applicationin semiconductor manufacturing, 2009 International Conference on Computers and Industrial Engineering, CIE 2009. IEEE Computer Society, Troyes, France, pp. 211-216.

Graves, S., Meal, H., Stefek, D., Zeghmi, A., 1983. Scheduling of re-entrant flow shops. J. Oper. Manag. 3, 197-207.

Gupta, J., Stafford, E., 2006. Flowshop scheduling research after five decades. Euro. J. Oper. Res.169, 699-711.

Haitham, H., Ruml, W., 2006. Network flow modeling for flexible manufacturing systems with re-entrant lines, Proceedings of the 45th IEEE Conference on Decision and Control IEEE, Piscataway, NJ, USA, p. 8 pp. 
Hwang, H., Sun, J.U., 1998. Production sequencing problem with re-entrant work flows and sequence dependent setup times. Int. J. Prod. Res. 36, 2435-2450.

Janiak, A., 1988. General flow-shop scheduling with resource constraints. Int. J. Prod. Res. 26, 1089-1103.

Jiang, S., Tang, L., 2008. Lagrangian relaxation algorithms for re-entrant hybrid flowshop scheduling, 2008 International Conference on Information Management, Innovation Management and Industrial Engineering. IEEE, Piscataway, NJ, USA, pp. 78-81.

Kaihara, T., Fujii, N., Tsujibe, A., Nonaka, Y., 2010. Proactive maintenance scheduling in a re-entrant flow shop using Lagrangian decomposition coordination method. CIRP Ann. - Manuf. Technol. 59, 453-456.

Kolisch, R., Sprecher, A., 1997. PSPLIB-a project scheduling problem library. Euro. J. Oper. Res.96, 205-216.

Lee, C.K.M., Lin, D., 2010. Hybrid genetic algorithm for bi-objective flow shop scheduling problems with re-entrant jobs, IEEE International Conference on Industrial Engineering and Engineering Management, IEEM2010. IEEE Computer Society, Macao, China, pp. 1240-1245.

Leu, S.-S., Hwang, S.-T., 2002. GA-based resource-constrained flow-shop scheduling model for mixed precast production. Auto. in Constr. 11, 439-452.

Lin, D., Lee, C.K.M., 2010. A review of the research methodology for the re-entrant scheduling problem. Int. J. Prod. Res. 49, 2221-2242.

Liu, C.-H., 2010. A genetic algorithm based approach for scheduling of jobs containing multiple orders in a three-machine flowshop. Int. J. Prod. Res. 48, 4379-4396.

Nawaz, M., Enscore Jr, E.E., Ham, I., 1983. A heuristic algorithm for the m-machine, njob flow-shop sequencing problem. Omega 11, 91-95.

Neubert, G., Savino, M.M., 2009. Flow shop operator scheduling through constraint satisfaction and constraint optimisation techniques. Int. J. Prod. and Qual. Manag. 4, 549-568. 
Nielsen, E.H., 2004. How does variability in input load relate to the probability of critically delayed delivery in a simple multipart re-entrant flow-line problem? Int. J. Prod. Res. 42, 3383-3396.

Odrey, N.G., Green, J.D., Appello, A., 2001. A generalized Petri net modeling approach for the control of re-entrant flow semiconductor wafer fabrication. Robot. and Comput.Integr. Manuf.17, 5-11.

Pan, J.H., Chen, J.S., 2003. Minimizing makespan in re-entrant permutation flow-shops. J. Oper. Res. Soc. 54, 642-653.

Park, Y., Kim, S., Jun, C.H., 2000. Performance analysis of re-entrant flow shop with single-job and batch machines using mean value analysis. Prod. Plan. and Control 11, 537-546.

Perkins, J.R., Kumar, P.R., 1995. Optimal control of pull manufacturing systems. IEEE Trans. Autom. Control 40, 2040-2051.

Rau, H., Cho, K.-H., 2009. Genetic algorithm modeling for the inspection allocation in reentrant production systems. Expert Syst.with Appl. 36, 11287-11295.

Scholz-Reiter, B., Heger, J., Hildebrandt, T., 2010. Analysis of priority rule-based scheduling in dual-resource-constrained shop-floor scenarios, International Conference on Advances in Machine Learning and Systems Engineering. Springer Verlag, Berkeley, CA, United states, pp. 269-281.

Topcuoglu, H.R., Demiroz, B., Kandemir, M., 2007. Solving the register allocation problem for embedded systems using a hybrid evolutionary algorithm. IEEE Trans. Evol. Comput. 11, 620-634. 


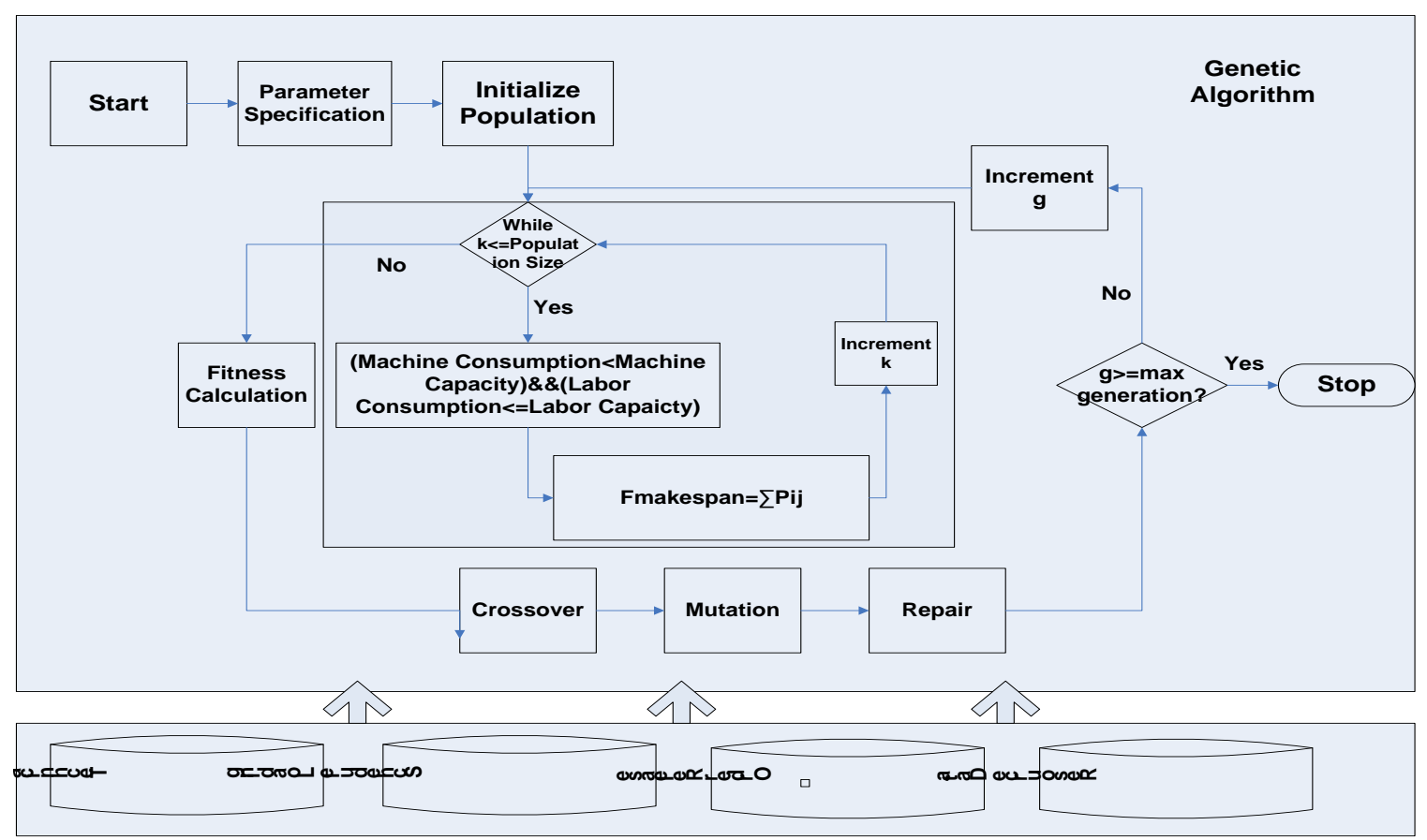

Fig. 1. Framework of multi-level GA for resource constrained scheduling. 


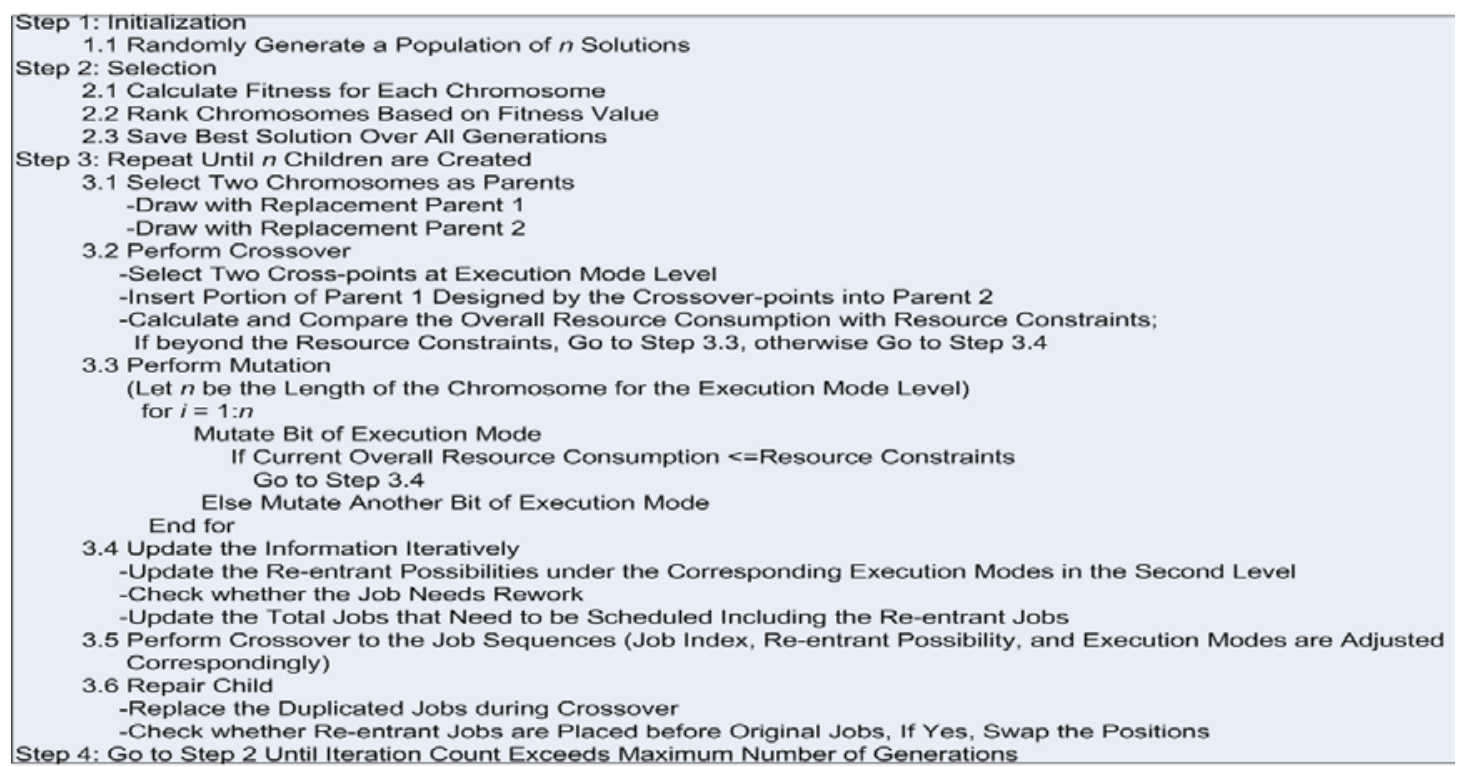

Fig. 2. Pseudocode for the GA. 

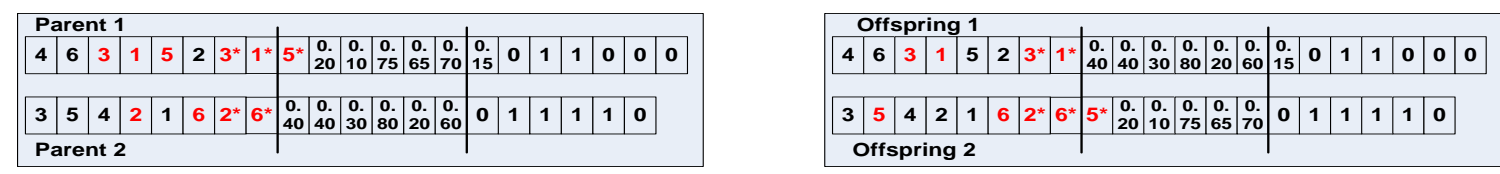

One-level encoding

\begin{tabular}{|c|c|c|c|c|c|c|c|c|}
\hline \multicolumn{9}{|c|}{ Parent 1} \\
\hline 4 & 6 & 3 & 1 & 5 & 2 & 3* & $1^{*}$ & 5* \\
\hline 0.20 & 0.10 & 0.75 & 0.65 & 0.70 & 0.15 & & & \\
\hline 0 & 1 & 1 & 0 & 0 & 0 & & & \\
\hline \multicolumn{2}{|c|}{ Parent 2} & & & & & & & \\
\hline 3 & 5 & 4 & 2 & 1 & 6 & $2 *$ & 6* & \\
\hline 0.40 & 0.40 & 0.30 & 0.80 & 0.20 & 0.60 & & & \\
\hline 0 & 1 & 1 & 1 & 1 & 0 & & & \\
\hline
\end{tabular}

\begin{tabular}{|c|c|c|c|c|c|c|c|c|}
\hline \multirow[b]{2}{*}{ Job Index } & \multicolumn{8}{|c|}{ Offspring 1} \\
\hline & 4 & 6 & 3 & 1 & 5 & 2 & $3 *$ & $5 *$ \\
\hline $\begin{array}{l}\text { Re-entrant } \\
\text { Possibility }\end{array}$ & 0.20 & 0.10 & 0.75 & 0.20 & 0.70 & 0.15 & & \\
\hline \multirow[t]{2}{*}{$\begin{array}{l}\text { Execution } \\
\text { Mode }\end{array}$} & $\mathbf{0}$ & 1 & 1 & 1 & 0 & $\mathbf{0}$ & & \\
\hline & \multicolumn{2}{|c|}{ Offspring } & \multicolumn{2}{|l|}{2} & & & & \\
\hline Job Index & 3 & 5 & 4 & 2 & 1 & 6 & & \\
\hline $\begin{array}{l}\text { Re-entrant } \\
\text { Possibility }\end{array}$ & \multicolumn{2}{|c|}{\begin{tabular}{|l|l|}
0.40 & $0.4 \mathrm{Q}$ \\
\end{tabular}} & \multicolumn{2}{|c|}{\begin{tabular}{|l|l|}
0.30 & 0.15 \\
\end{tabular}} & \multicolumn{2}{|c|}{\begin{tabular}{|l|l|}
0.10 & 0.15 \\
\end{tabular}} & & \\
\hline $\begin{array}{l}\text { Execution } \\
\text { Mode }\end{array}$ & $\mathbf{0}$ & 1 & 1 & $\mathbf{0}$ & 1 & 0 & & \\
\hline
\end{tabular}

Multi-level encoding

Fig. 3. One-level encoding vs. Multi-level encoding. 


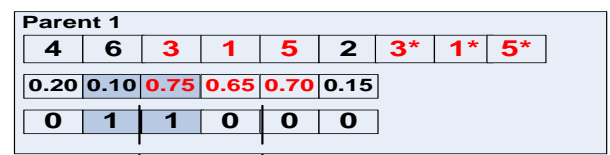

Original parents

\begin{tabular}{|c|c|c|c|c|c|c|c|c|}
\hline 4 & 6 & 3 & 1 & 5 & 2 & $3^{*}$ & $1^{*}$ & $5^{*}$ \\
\hline 0.20 & 0.10 & 0.75 & 0.65 & 0.70 & 0.15 \\
\hline 0 & 1 & 1 & 1 & 0 & 0 \\
\hline
\end{tabular}

Step 1-crossover at execution mode

\begin{tabular}{|l|l|l|l|l|l|l|l|l|}
\hline 4 & 6 & 3 & 1 & 5 & 2 & $3^{*}$ & $1^{*}$ & * \\
\hline
\end{tabular}

\begin{tabular}{|l|l|l|l|l|l|}
\hline 0.20 & 0.10 & 0.75 & 0.20 & 0.70 & 0.15 \\
\hline
\end{tabular}

\begin{tabular}{|l|l|l|l|l|l|}
\hline $\mathbf{0}$ & $\mathbf{1}$ & $\mathbf{1}$ & $\mathbf{1}$ & $\mathbf{0}$ & $\mathbf{0}$ \\
\hline
\end{tabular}

Step 2-update the re-entrant possibility Offspring 1

\begin{tabular}{|l|l|l|l|l|l|l|l|}
\hline 4 & 6 & 3 & 1 & 5 & 2 & $3^{*}$ & $5^{*}$ \\
\hline
\end{tabular}

\begin{tabular}{|l|l|l|l|l|l|l|}
\hline $\mathbf{0}$ & 0.20 & 0.10 & 0.75 & 0.20 & 0.70 & 0.15 \\
\hline
\end{tabular}

\begin{tabular}{|l|l|l|l|l|l|}
\hline $\mathbf{0}$ & $\mathbf{1}$ & $\mathbf{1}$ & $\mathbf{1}$ & $\mathbf{0}$ & $\mathbf{0}$ \\
\hline
\end{tabular}

Step 3-new offspring

Fig. 4. Crossover procedure.

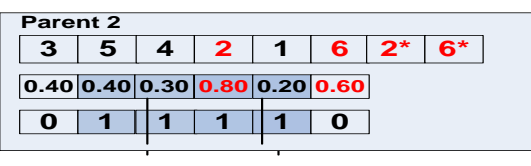

\begin{tabular}{|l|l|l|l|l|l|l|l|}
\hline 3 & 5 & 4 & 2 & 1 & 6 & $2^{*}$ & $6^{*}$ \\
\hline
\end{tabular} \begin{tabular}{|l|l|l|l|l|l|l|l|}
\hline 0.40 & 0.40 & 0.30 & 0.80 & 0.20 & 0.60 \\
\hline
\end{tabular} \begin{tabular}{|l|l|l|l|l|l|}
\hline $\mathbf{0}$ & $\mathbf{1}$ & $\mathbf{1}$ & $\mathbf{0}$ & $\mathbf{1}$ & $\mathbf{0}$ \\
\hline
\end{tabular}

\begin{tabular}{|l|l|l|l|l|l|l|l|}
\hline 3 & 5 & 4 & 2 & 1 & 6 & $2^{*}$ & $6^{*}$ \\
\hline
\end{tabular} \begin{tabular}{|l|l|l|l|l|l|l|l|}
\hline 0.40 & 0.40 & 0.30 & 0.15 & 0.10 & 0.15 \\
\hline
\end{tabular} \begin{tabular}{|l|l|l|l|l|l|}
\hline $\mathbf{0}$ & $\mathbf{1}$ & $\mathbf{1}$ & $\mathbf{0}$ & $\mathbf{1}$ & $\mathbf{0}$ \\
\hline
\end{tabular}

Offspring 2
\begin{tabular}{|c|c|c|c|c|c|}
\hline 3 & 5 & 4 & 2 & 1 & 6 \\
\hline 0.40 & 0.40 & 0.30 & 0.15 & 0.10 & 0.15 \\
\hline 0 & 1 & 1 & 0 & 1 & 0 \\
\hline
\end{tabular}


Table 1 Parameter setting for GA and SA

\begin{tabular}{|c|c|c|c|}
\hline \multicolumn{2}{|c|}{ Genetic algorithm (without tuning) } & \multicolumn{2}{|c|}{ Simulated annealing (with tuning) } \\
\hline Parameter & Value & Parameter & Value \\
\hline Population size & 200 & Stopping criteria & 200 generation \\
\hline Fitness scaling & Rank & Acceptance criteria & SA acceptance \\
\hline $\begin{array}{l}\text { Chromosome } \\
\text { representation }\end{array}$ & $\begin{array}{l}\text { Three level real } \\
\text { problem encoding }\end{array}$ & Annealing function & Fast annealing \\
\hline Crossover operator & Two-point & $\begin{array}{l}\text { Problem } \\
\text { representation }\end{array}$ & $\begin{array}{l}\text { Variable-length } \\
\text { encoding }\end{array}$ \\
\hline Mutation operator & $\begin{array}{l}\text { Gaussian } \\
(\text { scale }=1.5, \\
\text { shrink=1) }\end{array}$ & $\begin{array}{l}\text { Number of runs per } \\
\text { problem size }\end{array}$ & 10 runs \\
\hline $\begin{array}{l}\text { Crossover } \\
\text { probability }\end{array}$ & 0.8 & Starting point & {$\left[\begin{array}{lllll}0 & 0 & 0 & \ldots & 0\end{array}\right]$} \\
\hline Mutation probability & 0.4 & & \\
\hline Stopping criteria & 100 generation & & \\
\hline Parent selection & Uniform selection & & \\
\hline $\begin{array}{l}\text { Number of runs per } \\
\text { problem size }\end{array}$ & 10 runs & & \\
\hline
\end{tabular}


Table 2 Average performance of GA and SA without GA parameter tuning

\begin{tabular}{|c|c|c|c|c|c|c|c|c|}
\hline \multirow{2}{*}{$\begin{array}{l}\text { Job } \\
\text { size }\end{array}$} & \multirow{2}{*}{$\begin{array}{l}\text { Data } \\
\text { source }\end{array}$} & \multirow{2}{*}{$\begin{array}{l}\text { Data } \\
\text { set }\end{array}$} & \multicolumn{3}{|l|}{$\mathrm{GA}$} & \multicolumn{3}{|l|}{ SA } \\
\hline & & & Mean & $\begin{array}{l}\text { St. } \\
\text { deviation }\end{array}$ & Best & Mean & $\begin{array}{l}\text { St. } \\
\text { deviation }\end{array}$ & Best \\
\hline \multirow{4}{*}{$\begin{array}{l}20 \\
\text { jobs }\end{array}$} & C1563_7 & Set 1 & 958.0648 & 25.71703 & 906.969 & 971.5597 & 23.27611 & 940.78 \\
\hline & C1563_7 & Set 2 & 990.4381 & 27.3935 & 933.85 & 994.3035 & 26.42774 & 946.12 \\
\hline & C1563_8 & Set 3 & 1015.614 & 30.8407 & 957.22 & 1034.202 & 23.09262 & 993.30 \\
\hline & C1563_8 & Set 4 & 1157.2 & 28.31164 & 1104.36 & 1158.831 & 25.74849 & 1116.4 \\
\hline \multirow{4}{*}{$\begin{array}{l}30 \\
\text { jobs }\end{array}$} & C1563-7 & Set 1 & 1588.413 & 23.4718 & 1548.97 & 1606.738 & 26.82226 & 1548.269 \\
\hline & C1554_10 & Set 2 & 1451.745 & 26.707 & 1394.879 & 1462.596 & 32.23332 & 1403.929 \\
\hline & C1563_8 & Set 3 & 1580.758 & 34.04155 & 1518.86 & 1598.082 & 46.03813 & 1513.42 \\
\hline & C1562_3 & Set 4 & 1602.434 & 43.05558 & 1517.26 & 1631.362 & 42.59203 & 1555.04 \\
\hline \multirow{4}{*}{$\begin{array}{l}40 \\
\text { jobs }\end{array}$} & C1563_7 & Set 1 & 1988.06 & 40.62337 & 1910.7 & 2021.577 & 43.99321 & 1932.029 \\
\hline & C1563_8 & Set 2 & 2202.546 & 57.62981 & 2100.459 & 2224.944 & 77.26172 & 2103.00 \\
\hline & C1563_5 & Set 3 & 2004.31 & 38.92844 & 1932.4 & 2044.212 & 99.75263 & 1933.769 \\
\hline & C1563_4 & Set 4 & 1900.255 & 30.94322 & 1857.5 & 1953.697 & 41.13965 & 1862.429 \\
\hline
\end{tabular}


Table 3 Tested parameters for GA

\begin{tabular}{|l|l|}
\hline Parameters & Levels \\
\hline Population sizes & $100 \sim 200$, in steps of 10 \\
\hline Crossover types & Heuristic, two-point \\
\hline Mutation types & Gaussian, uniform \\
\hline Crossover rates & $0 \sim 1$, in steps of 0.05 \\
\hline Mutation rates & $0 \sim 0.2$, in steps of 0.01 \\
\hline
\end{tabular}


Table 4 Experimental factors

\begin{tabular}{|c|c|c|c|c|c|c|c|}
\hline \multirow{2}{*}{ Factors } & Levels & \multicolumn{6}{|c|}{ Values } \\
\cline { 3 - 8 } & \multicolumn{2}{|c|}{20 jobs } & \multicolumn{2}{c|}{30 jobs } & \multicolumn{2}{c|}{40 jobs } \\
\cline { 3 - 8 } & High & Low & High & Low & High & Low \\
\hline $\begin{array}{c}\text { Population } \\
\text { size (A) }\end{array}$ & 2 & 190 & 110 & 190 & 170 & 190 & 130 \\
\hline $\begin{array}{c}\text { Crossover } \\
\text { rate (B) }\end{array}$ & 2 & 0.5 & 0.1 & 0.6 & 0.45 & 0.65 & 0.4 \\
\hline $\begin{array}{c}\text { Mutation } \\
\text { rate (C) }\end{array}$ & 2 & 0.1 & 0.03 & 0.14 & 0.05 & 0.12 & 0.03 \\
\hline
\end{tabular}




\section{Table 5 ANOVA results}

\begin{tabular}{|l|l|l|l|}
\hline \multirow{2}{*}{ Factor } & \multicolumn{3}{c|}{$\mathbf{F}_{\mathbf{0}}$ value } \\
\cline { 2 - 4 } & \multicolumn{1}{|c|}{20 jobs } & \multicolumn{1}{c|}{30 jobs } & \multicolumn{1}{c|}{40 jobs } \\
\hline$A$ & 12.91331 & 12.17451 & 12.69831 \\
\hline$B$ & 0.682741 & 0.145436 & 0 \\
\hline$C$ & 0.34964 & 0.022919 & 5.150436 \\
\hline$A B$ & 2.051761 & 0.145436 & 0 \\
\hline$A C$ & 2.630544 & 0.835599 & 0.315796 \\
\hline$B C$ & 2.05922 & 0.145436 & 0 \\
\hline$A B C$ & 0.701718 & 0.145436 & 0 \\
\hline
\end{tabular}


Table 6 Comparisons of our GA with SA for larger problem sizes

\begin{tabular}{|c|c|c|c|c|c|}
\hline \multirow[t]{2}{*}{ \# } & \multirow{2}{*}{$\begin{array}{c}\text { Data } \\
\text { source }\end{array}$} & \multirow{2}{*}{$\begin{array}{l}\text { Resource } \\
\text { constraint }\end{array}$} & \multicolumn{2}{|c|}{ Makespan } & \multirow{2}{*}{$\frac{S A-G A}{G A}$} \\
\hline & & & GA & SA & \\
\hline \multirow[t]{4}{*}{50 jobs } & \multirow[t]{4}{*}{ C1562_9 } & Scenario 1 & 2614.5 & 2601.19 & $-0.509 \%$ \\
\hline & & Scenario 2 & 2449.3 & 2651.45 & $8.25 \%$ \\
\hline & & Scenario 3 & 2606.1 & 2580.81 & $-0.9 \%$ \\
\hline & & Scenario 4 & 2538.9 & 2600.22 & $2.42 \%$ \\
\hline \multirow[t]{4}{*}{100 jobs } & \multirow{4}{*}{$\begin{array}{l}\text { C1564_7, } \\
\text { C1564_8 }\end{array}$} & Scenario 1 & 4857 & 4961.32 & $2.15 \%$ \\
\hline & & Scenario 2 & 4909.7 & 4867.16 & $-0.87 \%$ \\
\hline & & Scenario 3 & 4874.9 & 4965.36 & $1.96 \%$ \\
\hline & & Scenario 4 & 4904.4 & 4959.43 & $1.12 \%$ \\
\hline \multirow[t]{4}{*}{150 jobs } & \multirow{4}{*}{$\begin{array}{l}\text { C1564_6, } \\
\text { C1564_9, } \\
\text { C1564_10 }\end{array}$} & Scenario 1 & 8362.97 & 8505.19 & $1.7 \%$ \\
\hline & & Scenario 2 & 8506.23 & 8567.12 & $0.72 \%$ \\
\hline & & Scenario 3 & 8453.6 & 8538.35 & $1.00 \%$ \\
\hline & & Scenario 4 & 8293.3 & 8514.3 & $2.67 \%$ \\
\hline \multirow[t]{4}{*}{200 jobs } & \multirow{4}{*}{$\begin{array}{l}\text { C1564_1, } \\
\text { C1564_2, } \\
\text { C1564_3, } \\
\text { C1564_4, } \\
\text { C1564_5 }\end{array}$} & Scenario 1 & 11073.35 & 11260.12 & $1.66 \%$ \\
\hline & & Scenario 2 & 10971.21 & 11053.1 & $0.74 \%$ \\
\hline & & Scenario 3 & 11029.14 & 10818.57 & $-1.59 \%$ \\
\hline & & Scenario 4 & 11006.31 & 11104.01 & $0.88 \%$ \\
\hline
\end{tabular}




\section{University Library}

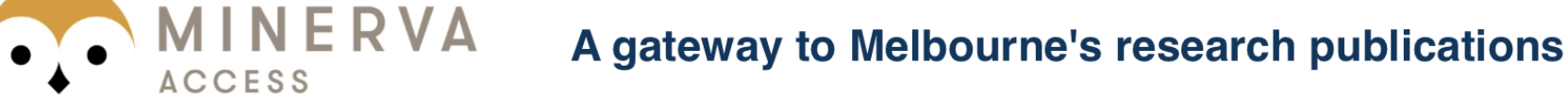

Minerva Access is the Institutional Repository of The University of Melbourne

Author/s:

Lin, D;Lee, CKM;Ho, W

Title:

Multi-level genetic algorithm for the resource-constrained re-entrant scheduling problem in the flow shop

Date:

2013

Citation:

Lin, D., Lee, C. K. M. \& Ho, W. (2013). Multi-level genetic algorithm for the resource-

constrained re-entrant scheduling problem in the flow shop. Engineering Applications of Artificial Intelligence, 26 (4), pp.1282-1290. https://doi.org/10.1016/j.engappai.2012.10.006.

Persistent Link:

http://hdl.handle.net/11343/118655 